\title{
Association of coronary ischemia estimated by fractional flow reserve and psychological characteristics of patients
}

\author{
Miodrag Jovan Sreckovic ${ }^{1}$, Nikola Jagic ${ }^{2}$, Vladimir Miloradovic ${ }^{1}$, Aleksandar Neskovic ${ }^{3}$, Ivan Soldatovic ${ }^{4}$, \\ Ilija Srdanovic ${ }^{5,6}$
}

${ }^{1}$ Department of Internal Medicine, Faculty of Medical Sciences, University of Kragujevac, Serbia

${ }^{2}$ Department of Radiology, Faculty of Medical Sciences, University of Kragujevac, Serbia

${ }^{3}$ Department of Cardiology, Clinical Hospital Centre Zemun, Faculty of Medicine, University of Belgrade, Belgrade, Serbia

${ }^{4}$ Institute for Biomedical Statistics, School of Medicine, University of Belgrade, Belgrade, Serbia

${ }^{5}$ Faculty of Medicine, University of Novi Sad, Novi Sad, Serbia

${ }^{6}$ Institute of Cardiovascular Diseases of Vojvodina, Clinic of Cardiology, Sremska Kamenica, Serbia

Adv Interv Cardiol 2017; 13, 2 (48): 117-121

DOI: https://doi.org/10.5114/pwki.2017.68009

\begin{abstract}
A bstract
Introduction: Psychological characteristics of patients, depression, stress and anxiety are recognized as important confounding risk factors for ischemic heart disease. However, the impact of psychological characteristics on coronary ischemia and vice versa remain poorly understood.

Aim: To demonstrate the interplay of psychological characteristics, depression, stress and anxiety with coronary ischemia estimated with fractional flow reserve (FFR).

Material and methods: From 2014 to 2016, 147 patients who were planned for FFR measurement were included in this study. Psychological characteristics of patients were evaluated using the Depression, Anxiety and Stress Scale 21 items (DASS 21) self-report questionnaire.

Results: Comparing the FFR ischemic vs. FFR non-ischemic groups, a significant difference was observed regarding results achieved for the depression, anxiety and stress scales. Multivariate logistic regression analysis was used to model the correlation between FFR and the DAS scale. It was clear, when controlling for previous myocardial infarction, that FFR was significant in all analyses. However, when the Canadian Cardiovascular Society grading of angina pectoris (CCS) class was entered in the model, FFR was not a significant predictor of anxiety, but was significant in other analysis.

Conclusions: Higher degrees of the psychological characteristics depression, stress and anxiety were observed in the group of patients with coronary ischemia, corresponding to lower fractional flow values.
\end{abstract}

Key words: fractional flow reserve, coronary ischemia, depression, stress, anxiety.

\section{Introduction}

Psychological characteristics of patients, predominantly depression, are recognized as an important, independent risk factor in cardiovascular diseases (CVD). Both diseases are leading causes of disability in developed countries and are expected to become so for countries of all income levels by 2030 [1]. The impact of depression as a cardiovascular risk factor was previously investigated and it is ranked in an intermediate position between the "big five" classical cardiovascular risk factors. In fact, it is more strongly associated with mortality than hypercholesterolemia and obesity [2]. Approximately $20 \%$ of patients with coronary heart disease (CHD) have major depression and $20 \%$ have minor depression at any given point in the course of their illness [3]. Depressed patients had higher rates of cardiac complications, including recurrent ischemia, infarction, or heart failure during their first hospital stay or readmission for angina, recurrent acute myocardial infarction (MI), chronic heart failure, or arrhythmias as compared to non-depressed patients [4]. Also, it is demonstrated in prior studies that symptoms of anxiety and depression are correlates of angina pectoris by recent history and an ischemia-positive treadmill test in patients with documented coronary artery disease (CAD) [5]. Depression is associated with angina, independently of CAD severity, due to which patients with depression may not

\section{Corresponding author:}

Miodrag Jovan Sreckovic MD, Department of Internal Medicine, Faculty of Medical Sciences, University of Kragujevac, $5 a / 4$ Kumanovska St, 34000 Kragujevac, Serbia, phone: +38 1693371777, e-mail: sreckovic7@gmail.com

Received: 9.03.2017, accepted: 28.04.2017. 
derive as much symptomatic benefit from revascularization as those without [6]. Endothelial function has been of interest as a possible mechanism linking depression to CVD, and various studies support the inverse correlation between depressed mood and endothelial function, as measured by flow-mediated dilation (FMD) [7]. The fractional flow reserve (FFR) is currently considered the gold standard for estimating the functional significance of coronary stenosis. To our knowledge, there has been no published study investigating the association between coronary ischemia confirmed by FFR and psychological disorders of patients.

\section{Aim}

Thus, the aim of our study is to assess the correlation between coronary ischemia, measured with FFR, and psychological characteristics of patients: depression, stress and anxiety.

\section{Material and methods}

One hundred forty-seven patients were enrolled in our research in three clinical centers and one hospital in Serbia, during March 2015 until April 2016. The research was approved by the Ethics Committees of these institutions and informed consent to participate in the study was obtained. A multicenter, cross-sectional analytical study was performed. Coronary artery lesions were evaluated using quantitative coronary analysis (QCA) and they were considered as intermediate if they were within the range 50-80\%. The study population consisted of patients for whom FFR measurement was planned to evaluate the ischemic potential of intermediate coronary lesions. Diagnostic coronary angiography and FFR measurements are routine diagnostic procedures, which are performed independently of the research. After coronarography was performed and intermediate lesions were observed using QCA, we obtained informed consent from patients to participate in our study. The participation of the patient in the study consists of filling in the self-report questionnaire; thus our study was observational by its nature, i.e. non-interventional. After patients were consented for participation, they were asked to answer the DASS 21 self-report questionnaire. The FFR measurement was performed after patients answered the DASS 21 questionnaire, to evaluate the ischemic potential of coronary lesions.

Estimation of depression, anxiety and stress symptoms was performed using the Serbian version of the DASS 21 self-report questionnaire. The DASS 21 is a set of three self-report scales designed to measure the negative emotional states of depression, anxiety and stress. Numerous scales for psychological evaluation of patients are available to researchers worldwide, most of which are used in the English speaking countries. Only a few of them have been translated into Serbian, and even fewer have been psychometrically evaluated in the Serbian population. DASS 21 is one of the most often used instruments for assessing unpleasant emotional states, symptoms of depression, anxiety and stress in the clinical and non-clinical population. It is characterized by excellent psychometric characteristics. The scale is in the public domain, and no additional charges are required for using it. DASS 21 was previously validated in the Serbian population. The results showed that the DASS 21 is a reliable and valid measure of unpleasant emotions [8].

\section{Statistical analysis}

Data are presented as counts (percentages) or mean \pm standard deviation or median $\left(24^{\text {th }}-75^{\text {th }}\right.$ percentile) depending on data type and distribution. The $\chi^{2}$ test, Mann-Whitney $U$ test and test were used to assess significant differences between groups. Multivariate logistic regression was used to assess the correlation between FFR and DAS scales when controlled for confounding factors. All $p$-values less than 0.05 were considered significant. All data were analyzed using SPSS 20.0 (IBM corp.) statistical software.

\section{Results}

The study included 147 patients, mean age 63.0 \pm 8.82 years, 109 (74.1\%) males. Of the total sample, 63 (42.8\%) patients had an FFR value $\leq 0.80$ and constituted the ischemic group, while 84 (57.1\%) patients had an FFR value $>0.80$ and constituted the non-ischemic group.

When we analyzed characteristics between the FFR-measured ischemic vs. non-ischemic group, there was a significant difference regarding CCS anginal class and previous myocardial infarction (Table I).

Comparing the FFR ischemic vs. FFR non-ischemic group, a significant difference was observed in results obtained for depression, anxiety and stress scales and in the percentage of coronary stenosis (Table II).

Multivariate logistic regression analysis was used to model the correlation between FFR and DAS scales. It was clear, when controlling for previous IM and gender, that FFR was significant in all analyses. However, when CCS class was entered in the model, FFR was not a significant predictor of anxiety, but was significant in other analysis (Table III). One can argue whether CCS class should be entered in the model, because it is a subjective measurement of patients' anginal symptoms while FFR is an objective measurement of coronary ischemia, so actually they both measure a similar entity. We also opted to enter gender in the multivariate logistic regression analysis model, since depression is much more common among women than men, and the majority of our study population consisted of men.

\section{Discussion}

Numerous studies conducted in the last decades have offered compelling evidence that depression is an 
Table I. Sociodemographic and clinical characteristics of patients

\begin{tabular}{|c|c|c|c|}
\hline \multirow[t]{2}{*}{ Parameter } & \multicolumn{2}{|c|}{ FFR } & \multirow[t]{2}{*}{$P$-value } \\
\hline & $\begin{array}{c}\text { Non- } \\
\text { ischemic }\end{array}$ & Ischemic & \\
\hline Age [years] & $62.4 \pm 9.6$ & $63.1 \pm 7.8$ & $0.660^{a}$ \\
\hline Sex male & $60(71.4 \%)$ & $49(77.8 \%)$ & $0.384^{b}$ \\
\hline Body mass index $\left[\mathrm{kg} / \mathrm{m}^{2}\right]$ & $28.3 \pm 3.5$ & $28.1 \pm 3.3$ & $0.749^{a}$ \\
\hline Non-smokers & $39(46.4 \%)$ & $25(39.7 \%)$ & $0.686^{b}$ \\
\hline Employed & $27(32.1 \%)$ & $16(25.4 \%)$ & $0.329^{b}$ \\
\hline Married & $69(82.1 \%)$ & $50(79.4 \%)$ & $0.671^{b}$ \\
\hline Hypertension & $84(100 \%)$ & $62(98.4 \%)$ & $0.429^{b}$ \\
\hline Diabetes mellitus & $27(32.1 \%)$ & $28(44.4 \%)$ & $0.127^{b}$ \\
\hline Previous MI & $24(28.6 \%)$ & $31(49.2 \%)$ & $0.011^{b}$ \\
\hline Coronary artery stenosis (\%) & $63.3 \pm 6.8$ & $70.2 \pm 9.2$ & $<0.001^{a}$ \\
\hline \multicolumn{4}{|l|}{ CCS class: } \\
\hline 0 & $16(20.8 \%)$ & $3(6.4 \%)$ & $0.001^{b}$ \\
\hline I & $23(29.9 \%)$ & $9(19.9 \%)$ & \\
\hline$\|$ & $26(33.8 \%)$ & $17(36.2 \%)$ & \\
\hline III & $11(14.3 \%)$ & $16(34.0 \%)$ & \\
\hline IV & $1(1.3 \%)$ & $2(4.3 \%)$ & \\
\hline
\end{tabular}

Results are presented as mean \pm standard deviations or counts (percents); ${ }^{a} t$ test, ${ }^{b} \chi^{2}$-square test.

independent risk factor for development of $\mathrm{CHD}$ and for worsening prognosis when CHD is present. Previous studies emphasized the strong influence of psychosocial factors on the patient's quality of life and adherence to therapy [9]. Persons with depression are more likely to eventually develop CVD and also have a higher mortality rate than the general population. Patients with CVD who
Table II. Depression, anxiety and stress

\begin{tabular}{lccr} 
& \multicolumn{2}{c}{ FFR } & P-value \\
\cline { 2 - 3 } & Non-ischemic & Ischemic & \\
\hline Depression score & $2(1-3)$ & $4(2-6)$ & $<0.001^{\mathrm{a}}$ \\
\hline Anxiety score & $2(1-4)$ & $4(3-7)$ & $<0.001^{\mathrm{a}}$ \\
\hline Stress score & $4(2-6)$ & $8(8-10)$ & $<0.001^{\mathrm{a}}$ \\
\hline Depression & $12(14.3 \%)$ & $24(38.1 \%)$ & $0.001^{\mathrm{b}}$ \\
\hline Anxiety & $28(33.3 \%)$ & $35(55.6 \%)$ & $0.007^{\mathrm{b}}$ \\
\hline Stress & $16(19.0 \%)$ & $36(57.1 \%)$ & $<0.001^{\mathrm{b}}$
\end{tabular}

${ }^{a}$ Mann-Whitney $\cup$ test, ${ }^{b} \chi^{2}$ test.

are also depressed have a worse outcome than those patients who are not depressed. A causal relationship with either CVD causing more depression or depression causing more CVD and a worse prognosis for CVD is probable [10]. An association of depression with mortality in CHD patients has often been reported, but only a few studies have examined the role of the co-occurrence of depression and anxiety, although extensive comorbidity is observed between depression and anxiety [11]. Chronic anxiety is associated with numerous pathophysiological processes, including increased sympathetic nervous system activity, inflammation, and hypertension [12]. A study conducted by Watkins et al. showed that increased mortality risk was most pronounced in the patient group with comorbid anxiety and depression, in whom the risk of mortality was 3-fold higher than in patients with neither anxiety nor depression. Those with elevated anxiety symptoms showed a 2-fold increased risk of mortality, suggesting that anxiety has an explanatory role that may be clinically important in the identification of patients at higher risk of mortality [13].

Various biological mechanisms have been proposed to explain the relationship between depression and anx-

Table III. Multivariate logistic regression model

\begin{tabular}{|c|c|c|c|c|c|c|}
\hline \multirow[t]{2}{*}{ Variable } & \multicolumn{2}{|c|}{ Depression } & \multicolumn{2}{|r|}{ Anxiety } & \multicolumn{2}{|r|}{ Stress } \\
\hline & $P$-value & OR $(95 \% \mathrm{Cl})$ & $P$-value & OR $(95 \% \mathrm{Cl})$ & $P$-value & OR $(95 \% \mathrm{Cl})$ \\
\hline \multicolumn{7}{|l|}{ Model 1: } \\
\hline FFR & 0.002 & $3.725(1.640-8.460)$ & 0.008 & $2.595(1.284-5.247)$ & $<0.001$ & $6.158(2.852-13.293)$ \\
\hline $\begin{array}{l}\text { Previous myocardial } \\
\text { infarction }\end{array}$ & 0.646 & $1.212(0.534-2.750)$ & 0.600 & $1.213(0.589-2.496)$ & 0.365 & $0.693(0.314-1.531)$ \\
\hline Sex female & 0.181 & $1.810(0.759-4.317)$ & 0.027 & $2.408(1.106-5.241)$ & 0.876 & $0.935(0.400-2.183)$ \\
\hline \multicolumn{7}{|l|}{ Model 2: } \\
\hline FFR & 0.038 & $2.600(1.053-6.420)$ & 0.210 & $1.628(0.760-3.488)$ & $<0.001$ & $5.176(2.227-12.030)$ \\
\hline $\begin{array}{l}\text { Previous myocardial } \\
\text { infarction }\end{array}$ & 0.883 & $0.934(0.374-2.328)$ & 0.926 & $0.964(0.442-2.101)$ & 0.174 & $0.542(0.224-1.309)$ \\
\hline CCS class & $<0.001$ & $0.159(0.063-0.400)$ & 0.003 & $0.256(0.104-0.627)$ & 0.001 & $0.204(0.080-0.524)$ \\
\hline Sex female & 0.933 & $0.958(0.354-2.596)$ & 0.201 & $1.728(0.747-3.997)$ & 0.353 & $0.632(0.240-1.665)$ \\
\hline
\end{tabular}


iety and increased CVD risk. These include alterations in the autonomic nervous system [14], modification of platelet receptors and function [15], elevated pro-inflammatory cytokines, fibrinogen and plasminogen activator inhibitor-1 level [16]. Endothelial function was a point of interest also and diverse studies support the correlation between depressed mood and endothelial function impairment, measured by FMD [17].

In a study conducted by Ketterer et al., the association of specific psychological characteristics in patients having stable coronary disease with the reporting of anginal symptoms and positive exercise testing was investigated. Investigators reported that anxiety and depression were strong correlates of recent angina symptoms during daily activities and with angina in the presence of ischemia provoked by treadmill testing [5].

Recently published research performed by Hayek et al. examined whether depressive symptoms are associated with chest pain independently of coronary artery disease (CAD) severity, whether improvement in depressive symptoms over time is associated with improvement in chest pain and whether the impact of revascularization on chest pain differs between patients with and without depression [6]. They included 5158 patients in the research and reported that depression is associated with angina, independently of CAD severity. Also, patients with depression who underwent revascularization had less improvement in chest pain frequency than those without depressive symptoms [6].

A limitation of the study performed by Hayek et al. were the use of only coronary anatomy visual estimation for assessment of coronary lesion ischemic potential. The treadmill stress test used in the study by Ketterer et al. also have lower specificity and sensitivity than FFR measurement in coronary ischemia detection. To the best of our knowledge, our study is the first to have correlated FFR measurement as the current gold standard for evaluation of coronary ischemia and psychological characteristics of patients. Our study showed an inverse correlation of FFR measurement and a higher level of psychological symptoms indicated by the DASS 21 score. Multivariate logistic regression analysis was used to model the correlation between FFR and DAS scales. It was demonstrated when controlling for previous myocardial infarction and gender that FFR is significant in all the analysis. But when CCS class was entered in the model, FFR was not a significant predictor of anxiety, but was significant in other analysis. CCS class represents a patient's subjective evaluation of their anginal symptoms. It represents somatic hypersensitivity, a common manifestation of depression and anxiety. In research by Ketterer et al., patients who developed angina on a positive treadmill test also displayed higher scores on the Beck Depression Inventory $(p=0.003)$ and State Anxiety ( $p=0.004)$ scales [5]. Our result can raise the question whether somatic hypersensitivity is more influenced by depression than anxiety.
Since FFR measurement is not widely available, our suggestion for everyday practice when dealing with intermediate coronary lesions is to use self-report questionnaires such as the DASS 21 for initial patient screening and additional assessment. This self-report questionnaire is not time consuming, and we can use it to identify patients with a higher degree of psychological symptoms. Those patients require a multidisciplinary approach in their further treatment, and can benefit from comprehensive care.

It is still not known whether routine screening for psychosocial risk factors contributes to reducing future cardiovascular events. Socioeconomic status, social isolation and lack of social support are recognized in the latest European guidelines as risk modifiers that are likely to have reclassification potential when estimating cardiovascular risk [18].

Though European guidelines stated that clinical depression and depressive symptoms predict incident CAD (RR 1.6 and 1.9, respectively) [19], and worsen its prognosis (RR 1.6 and 2.4, respectively), routine assessment of psychosocial factors in patients and persons with CV risk factors is not mandatory. They should be considered for use as risk modifiers in CV risk prediction, especially in individuals with SCORE values near decisional thresholds [18].

On the other hand, in the National Heart Foundation of Australia position statement published in 2013, routine screening for depression in all patients with CHD is indicated at first presentation, and again at the next follow-up appointment. A follow-up screen should occur 2-3 months after a CHD event. Screening should then be considered on a yearly basis, as for any other major risk factor for CHD [20].

A limitation of our study is use of the DASS 21 self-report questionnaire to measure the severity of a range of depression, anxiety and stress symptoms. Although the DASS 21 may contribute to the diagnosis of depression and anxiety, it cannot replace a clinical interview.

\section{Conclusions}

Our study demonstrated an inverse correlation between fractional flow values used to evaluate coronary ischemia and DASS 21 results used to evaluate psychological characteristics of patients. In the group of patients with FFR-confirmed coronary ischemia, representing FFR measurement values less than 0.80 , elevated scores of psychological characteristics were present.

\section{Conflict of interest}

The authors declare no conflict of interest.

\section{References}

1. World Health Organization. The global burden of disease: 2004 update, 2008 Geneva, Switzerland. WHO Press. 
2. Ladwig KH, Baumert J, Marten-Mittag B, et al. Room for depressed and exhausted mood as a risk predictor for all-cause and cardiovascular mortality beyond the contribution of the classical somatic risk factors in men. Atherosclerosis 2017; 257: 224-31.

3. Carney RM, Freedland KE. Depression in patients with coronary heart disease. Am J Med 2008; 121 (11 Suppl. 2): S20-7.

4. Rumsfeld JS, Jones PG, Whooley $M$, et al. Depression predicts mortality and hospitalization in patients with myocardial infarction complicated by heart failure. Am Heart J 2005; 150: 961-7.

5. Ketterer MW, Bekkouche NS, Goldberg AD, et al. Symptoms of anxiety and depression are correlates of angina pectoris by recent history and an ischemia-positive treadmill test in patients with documented coronary artery disease in the Pimi Study. Cardiovasc Psych Neurol 2011; 2011: ID 134040.

6. Hayek SS, Ko YA, Awad M, et al. Depression and chest pain in patients with coronary artery disease. Int J Cardiol 2017; 230: 420-6.

7. Cooper DC, Tomfohr LM, Milic MS, et al. Depressed mood and flow-mediated dilation: a systematic review and meta-analysis. Psychosom Med 2011; 73: 360-9.

8. Jovanović V, Gavrilov-Jerković V, Žuljević D, et al. Psihometrijska evaluacija Skale depresivnosti, anksioznosti i stresa-21 (DASS21) na uzorku studenata u Srbiji. Psihologija 2014; 47: 93-112.

9. DiMatteo MR, Lepper HS, Croghan TW. Depression is a risk factor for noncompliance with medical treatment: meta-analysis of the effects of anxiety and depression on patient adherence. Arch Intern Med 2000; 160: 2101-7.

10. Hare DL, Toukhsati SR, Johansson P, et al. Depression and cardiovascular disease: a clinical review. Eur Heart J 2014; 35: 1365-72.

11. Kessler RC, Nelson CB, McGonagle KA, et al. Comorbidity of DSM-III-R major depressive disorder in the general population: results from the US National Comorbidity Survey. Br J Psychiatry Suppl 1996; 30: 17-30.

12. Pitsavos C, Panagiotakos DB, Papageorgiou C, et al. Anxiety in relation to inflammation and coagulation markers, among healthy adults: the ATTICA study. Atherosclerosis 2006; 185: 320-6.

13. Watkins LL, Koch GG, Sherwood A, et al. Association of anxiety and depression with all-cause mortality in individuals with coronary heart disease. J Am Heart Assoc 2013; 2: e000068.

14. de Jonge P, Mangano D, Whooley MA. Differential association of cognitive and somatic symptoms with heart rate variability in patients with stable coronary heart disease findings from the Heart and Soul study. Psychosom Med 2007; 69: 735-9.

15. Ziegelstein RC, Parakh K, Sakhuja A, Bhat U. Depression and coronary artery disease: is there a platelet link? Mayo Clin Proc 2007; 82: 1366-8.

16. Brouwers C, Mommersteeg PMC, Nykliček I, et al. Positive affect dimensions and their association with inflammatory biomarkers in patients with chronic heart failure. Biol Psychol 2013; 92: 220-6.

17. Cooper DC, Tomfohr LM, Milic MS, et al. Depressed mood and flow-mediated dilation: a systematic review and meta-analysis. Psychosom Med 2011; 73: 360-9.

18. Piepoli MF, Hoes AW, Agewall S, et al. 2016 European guidelines on cardiovascular disease prevention in clinical practice: the sixth joint task force of the European Society of Cardiology and other societies on cardiovascular disease prevention in clinical practice (constituted by representatives of 10 societies and by invited experts) developed with the special contribution of the European Association for Cardiovascular Prevention \& Rehabilitation (EACPR). Eur Heart J 2016; 37: 2315-81.
19. Spindler H, Pedersen SS. Posttraumatic stress disorder in the wake of heart disease: prevalence, risk factors, and future research directions. Psychosom Med 2005; 67: 715-23.

20. Colquhoun DM, Bunker SJ, Clarke DM, et al. Screening, referral and treatment for depression in patients with coronary heart disease. Med J Aust 2013; 198: 483-4. 